Acta Cryst. (2002). A58 (Supplement), C200

\section{CRYSTALLOGRAPHY FOR CHILDREN}

\section{K. M. Crennell}

Education officer, British Crystallographic Association

Very young children are intensely curious about their environment. By making them 'crystallographic play things' we can introduce them to concepts, which are essential to an understanding of the crystallography we hope they will study as adults. These are ideas of symmetry, space filling polyhedra, the lattice and the packing of spheres. Although children's building blocks in the UK are usually rectangular, other shapes can be made and used to discover by experiment which shapes completely fill space, a concept which later can lead to the idea of crystal classes. Young babies can learn from a 'Crystal Mobile of the Platonic Solids' hung over their cradle.

Crystal growing competitions are popular in the UK; while waiting for the crystals to grow children can be encouraged to make their own 3-D models from polyhedral nets. Older children can use the Internet to learn how crystals are important in our everyday lives by looking for links to topics such as 'How a quartz watch works' or how crystallography is used to improve magnetic materials in 'smart cards' or recording media on computer discs.

I am working on a set of web pages for the British Crystallographic Association incorporating these ideas and would be most grateful for suggestions of other ideas or links to educational websites.

\section{Keywords: EDUCATION CHILDREN 3D MODELS}

Acta Cryst. (2002). A58 (Supplement), C200

\section{STRUCTURAL STUDIES ON THE HUMAN ANDROGEN RECEPTOR} LIGAND-BINDING DOMAIN

M. A. Carrondo

Instituto de Tecnologia Quimica e Biologica, UNL, Oeiras, Portugal

Androgens and their receptors play an important role in male physiology and pathology. The human androgen receptor (hAR) binds the male sex steroids, and regulates genes for male differentiation and development. Therefore, mutations in the AR gene may lead to several disease states. The progression of human prostate cancer is often linked with a high level of AR expression or mutations. The knowledge of the 3-D structures of the AR receptor or specific mutants with natural or synthetic ligands are very important to the understanding of the interactions that may be involved with ligand specificity. The crystal structure of the hAR ligand-binding domain was solved in complex with the synthetic agonist metribolone, R1881, to $2.4 \AA$ resolution (Matias et al., JBC (2000)275, 26164-26171). The hAR double-mutant (L701H and T877A) has been reported to have high-affinity to cortisol/cortisone (Zhao et al., Nat.Med.(2000)6, 703-706). Its crystal structure was solved in complex with the agonist 9 - $\alpha$-fluorocortisol with a $1.95 \AA$ resolution (Matias et al., JMC (2001) in press). Both structures show the typical conformation of an agonistbound nuclear receptor in which Helix 12 is positioned as a lid for the ligandbinding pocket. Binding of 9- $\alpha$-fluorocortisol to the double-mutant involves favorable hydrogen bond patterns on the C17 and C21 substituents of the ligand due to the mutations at 701 and 877 in the AR. Our studies provide the first structural explanation for the glucocorticoid activation of AR mutant, which is important for the development of new therapeutic treatments for androgen-independent prostate cancer.

\section{Keywords: NUCLEAR RECEPTORS AGONIST LIGANDS ORISTATE} CANCER

\section{Acta Cryst. (2002). A58 (Supplement), C200}

CRYSTALLOGRAPHIC AND BIOCHEMICAL COMPARISON OF RXR HOMOAND HETERODIMERIZATION FUNCTIONS

W. Bourguet

Centre De Biochimie Structurale (CBS), CNRS/INSERM/UM 15 Avenue Charles

Flahault Montpellier 34060 FRANCE

Nuclear receptors (NRs) are ligand inducible transcription factors that regulate numerous processes involved in development, growth, cell differentiation, proliferation or apoptosis. All NRs are modular proteins that harbor one DNA-binding domain (DBD) and one ligand-binding domain (LBD). The LBD encodes a wealth of distinct functions, most of which operate in a ligand-dependent manner. The LBD harbors the ligand-binding pocket, the main dimerization surface and the ligand-dependent activation function AF-2. Detailed molecular insights into the structure-function relation of signal integration by NRs have been obtained by the elucidation of the crystal structures of several LBDs in different ligation states [reviewed in ref. 1].

Uniquely, the retinoid $\mathrm{X}$ receptors (RXRs) represent promiscuous dimerization partners for a large number of NRs including the retinoic acid receptors (RARs ) or the peroxisome proliferating activating receptors (PPARs). RXRs are also able to activate transcription from reporter genes as homodimers. However, previous studies have shown that the dissociation equilibrium constants (Kd) of heterodimers are more than two orders of magnitudes lower than the Kd for formation of RXR homodimers. RXR LBD as been crystallized in the homodimeric [2] as well as the RAR-RXR [3] and PPAR-RXR [4] heterodimeric forms. We will present a detailed comparative analysis of the homo- and heterodimerization interfaces of RXR LBD aimed at providing a rational for the higher potential of RXR to form heterodimers rather than homodimers. Our correlative crystallographic and mutational analysis reveals that to some extend, specificity of the residues involved in the formation of the RXR homo- and heterodimers can be identified and that RXR homo- and heterodimerization functions can be separated [5].

References

[1] Bourguet, W., Germain, P. and Gronemeyer, H. (2000). Trends in Pharmacological Sciences, 21, 381-388.

[2] Bourguet, W., Ruff, M., Chambon, P., Gronemeyer, H. and Moras, D. (1995). Nature, 375, 377-382.

[3] Bourguet, W., Vivat, V., Wurtz, J.M., Chambon, P., Gronemeyer, H. and Moras, D. (2000). Molecular Cell, 5, 289-298.

[4] Gampe RT, Montana VG, Lambert MH, Miller AB, Bledsoe RK, Milburn MV, Kliewer SA, Willson TM, Xu HE. (2000). Molecular Cell, 5, 545-555

[5]Vivat-Hannah, V., Bourguet, W., and Gronemeyer, H. (2002). Submitted.

\section{Acta Cryst. (2002). A58 (Supplement), C200}

THE PEROXISOME PROLIFERATOR-ACTIVATED RECEPTORS: STRUCTURAL INSIGHT INTO LIGAND-MEDIATED REGULATION E. Xu

GlaxoSmithKline Structural Sciences, Nuclear Receptor Discovery Research Structural Sciences V134, 5 Moore Dr., RTP, NC 27709 USA

The peroxisome proliferator-activated receptors (PPAR $\alpha, \gamma$ and $\delta$ ) are ligandactivated transcription factors that regulate glucose and fatty acid homeostasis and as such are exciting targets for the treatment of a variety of different metabolic diseases and cancers. We have determined the crystal structures of the ligand binding domains from all three PPARs, bound to agonists and antagonists, co-activators and co-repressors, and as monomers, homodimers and even heterodimers with RXR (retinoid X receptor). These structures have provided detail molecular insight into the ability of PPARs to bind diverse ligands, ranging from fibrates, and a class of lipid lowering drugs, to glitazones, a new generation of diabetes drugs. Structural comparisons between the agonist- and antagonist- bound PPARs highlight the important role of the C-terminal activation helix (AF-2) in recruitment of co-activators and co repressors that ultimately determine the receptor transcriptional functions. In this presentation, I will focus on key structural insights into the molecular basis of ligand binding and signaling by PPARs, with an emphasis on the conservation of these mechanisms applicable to the nuclear receptor superfamily

References

$\mathrm{Xu}$, et al, \& Stimmel. (2002) Nature, 415, 813-7.

Xu, Lambert et al, \&Willson. (2001) PNAS, 98. 13919-24

Gampe, et al, \& Xu. (2000) Mol. Cell, 5, 545-555

Gampe, et al, \& Xu. (2000) Genes \& Dev., 14, 2229-2239

Xu, et al, \& Milburn. (1999) Mol. Cell, 3, 397-403

Nolte, et al, \& Milburn. (1998) Nature, 395, 137-143

Keywords: NUCLEAR RECEPTORS, LIGANDS, COFACTORS 